\title{
Research and Application of Fiber Bragg Grating Sensor in Geological Disaster Automation Monitoring
}

\author{
Liu Yimin ${ }^{1,2}$, Wang $\mathrm{Jie}^{1}$, Ji Weifeng ${ }^{2}$, Zhou $\mathrm{Ce}^{2}$ and Chen Wenjun ${ }^{2}$ \\ ${ }^{1}$ School of Manufacturing Science \& Engineering, Sichuan University, Chengdu \\ China; ${ }^{2}$ The Institute of Exploration Technology of CAGS, Chengdu, China \\ liuyimin1005@gmail.com
}

\begin{abstract}
In this paper, fiber grating technology is used for accurately measure stresses, temperature and displacement. The paper introduces the basic principles of FBG technology and the wavelength demodulation method of FBG sensors, and put fiber grating technology into practical engineering projects. The paper design a optical fiber grating real-time automation monitoring system to distributed measure stress, temperature and displacement in geological disaster body, to provide effective technical supports for geological disaster prevention and mitigation.
\end{abstract}

Keywords: Fiber Bragg grating; Automation monitoring; Distributed measurement; Disaster prevention and mitigation

\section{Introduction}

In recent years, fiber grating communication and optical fiber sensing applications such as more and more attention. FBG sensing technology has become the optical fiber sensing technology in the most vigorous a technology. When directly on the core, small volume, has certain wavelength, Prague can reflect wavelength modulated physical quantities such as temperature, strain of characteristics, widely used in the optical fiber sensing.

As sensor component, fiber grating also possesses other special functions. For example, high ability of resisting electromagnetism disturb, small size and weight, high temperatureproof, high ability of multiplex, being liable to connect with fiber, low loss, good spectrum characteristic, erosion-proof, high sensitivity, being liable to deform and so on. At present, FBG (fiber Bragg grating) as sensor components has become the main stream of development and cultivation[1].

In the paper, the monitoring system take advantages of distributed FBG sensor network, wireless telemetry technology and geological disaster monitoring technology, and this system can real-time measure the stresses, temperature and displacement in landslide body.

The system has these following characteristics:

(1) The system realize remote multiple parameters network monitoring of landslide, because these feature of FBG sensor are anti-interference ability, highly precision and distributed measurement.

(2) According to characteristics of landslide monitoring and early warning, carrying out the design of network protocol and system software, and the system use GPRS, Beidou satellite and other ways for data transmission.

(3) According to the data of FBG sensors, we compare with various parameters and confirm the multi-azimuth monitoring, making contrast verification of these parameters, and at last supporting the formation of landslide monitoring and early warning model. 


\section{Structure of FBG Geological Disaster Automation Monitoring System}

The FBG monitoring system is made up of FBG sensing system, multiple channels wireless acquisition terminal and data publishing system, and this system can measure and monitor the stresses, temperature and displacement in real-time.

\subsection{System Principle}

A fiber Bragg grating (FBG) is a type of distributed Bragg reflector constructed in a short segment of optical fiber that reflects particular wavelengths of light and transmits all others. This is achieved by creating a periodic variation in the refractive index of the fiber core, which generates a wavelength specific dielectric mirror. As shown in Figure 2, a fiber Bragg grating can therefore be used as an inline optical filter to block certain wavelengths, or as a wavelength-specific reflector [1].
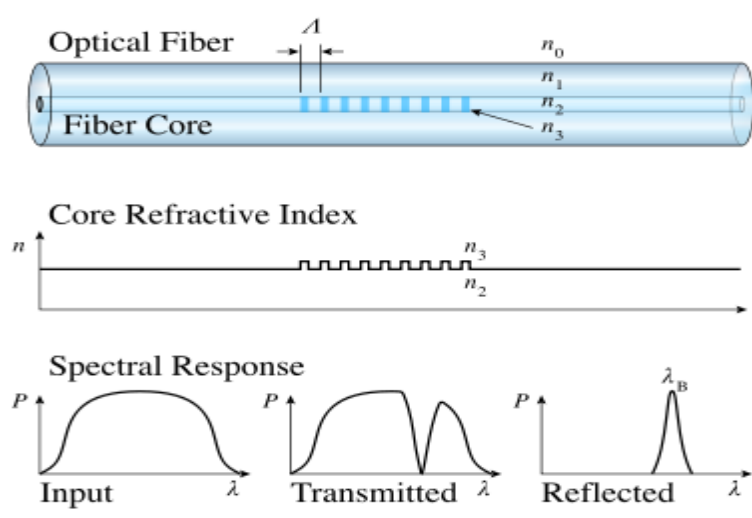

Figure 1. A Fiber Bragg Grating Structure with Refractive Index Profile and Spectral Response

The fundamental principle behind the operation of a fiber Bragg grating is Fresnel reflection, where light traveling between media of different refractive indices may both reflect and refract at the interface.

The refractive index will typically alternate over a defined length. The reflected wavelength $\left(\lambda_{B}\right)$, called the Bragg wavelength, is defined by the relationship[2].

$$
\lambda_{B}=2 n_{e} \Lambda
$$

Bragg grating Bragg reflection wavelength shift is influenced by temperature and stress[2]:

$$
\Delta \lambda_{\mathrm{B}} / \lambda_{\mathrm{B}}=\left(1-p_{e}\right) \varepsilon+(\alpha+\xi) \Delta T
$$

In this formula, ${ }^{p_{e}}$ is Valid elastic-optic coefficient, $\alpha$ is coefficient of thermal expansion,

$\xi$ is thermo-optic coefficient (dependent variable), $\Delta T$ is temperature changes. Based on these two kinds of effect, the Bragg grating can be used as sensitive element for strain and temperature measurement [2].

According to the basic working principle and demodulation methods of the fiber grating, the system accurately measure the wavelength of multiple parameters FBG sensors. The superiority of FBG sensor is that the sensing information is coded in the sensor wavelength. Using these measured wavelength, we can easily calculate the stress, displacement and 
temperature which we want to know[3]. According to these data changes comparison at different time, the parameter variation curve of each monitoring site is established.

Among them, interface of sensors wavelength measurements as shown in Figure 2.
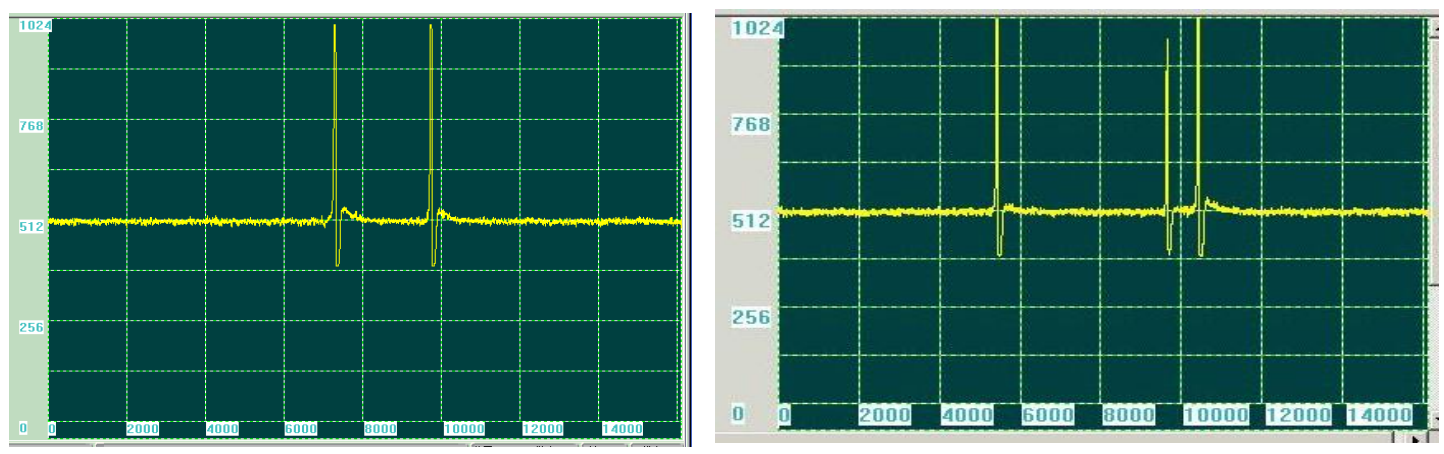

Figure 2. Wavelength Comparison with FBG Sensors and the Reference

Grating

(1) Stress measurement: because FBG precision is very high and temperature has a great influence on it, so two FBG sensor are needed for measurement. One is used for stress measurement, the other one is used for temperature compensation.

$$
P=K\left[\left(\lambda_{1}-\lambda_{0}\right)-\left(\lambda_{t 1}-\lambda_{t 0}\right)\right]
$$

Among them, $\mathrm{K}$ is coefficient of soil stress sensor, ${ }^{\lambda_{1}}$ is current value wavelength of this sensor, ${ }^{\lambda_{0}}$ is initial value wavelength of this sensor ; ${ }^{\lambda}$ is current value wavelength of temperature compensation sensor, ${ }^{\lambda_{t 0}}$ is initial value wavelength of temperature compensation sensor.

(2) Displacement measurement:the same as the measured stress, two FBG sensor are also needed for measurement.

$$
\Delta L=K\left[\left(\lambda_{1}-\lambda_{10}\right)-\left(\lambda_{2}-\lambda_{20}\right)\right]
$$

Among them, $K$ is coefficient of deformation sensor, ${ }^{\lambda_{1}}$ is current value wavelength of this sensor, ${ }^{\lambda}$ is initial value wavelength of this sensor ; ${ }^{\lambda}$ is current value wavelength of temperature compensation sensor, ${ }^{\lambda}{ }_{t 0}$ is initial value wavelength of temperature compensation sensor.

(3) Temperature measurement :

$$
T=K\left(\lambda-\lambda_{0}\right)+T_{0}
$$

Among them $K$ is temperature coefficient, ${ }^{\lambda_{1}}$ is current value wavelength of this sensor, $T$ is current temperature, ${ }^{\lambda_{0}}$ is the value wavelength when temperature is $T_{0}$, generally the wavelength takes $T=0^{\circ} \mathrm{C}$. 


\subsection{System Structure}

The system consists of FBG sensing system, multiple channels wireless acquisition terminal and data publishing system, structure chart of monitoring system as shown in Figure 3.

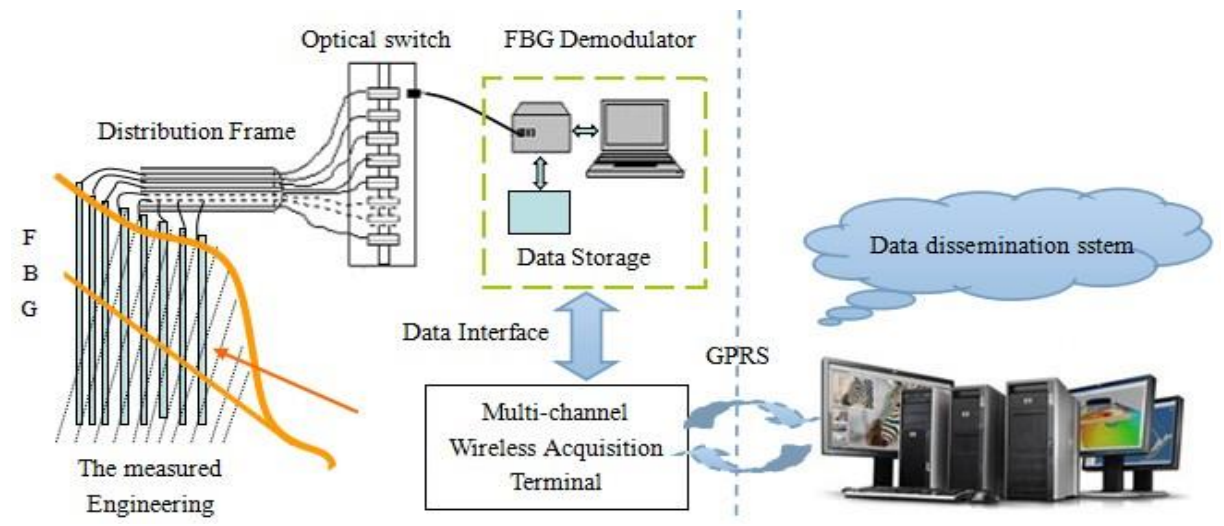

Figure 3. Structure Chart of Monitoring System

Among them, FBG sensing system measure by laying fiber Bragg grating sensor array on measurement pipe, and the sensor array consists of a set of fiber Bragg grating which packaging in accordance with the requirements for the measured object, and then, in tandem with single-mode fiber combined with reflection detection and F-P scanning optical filtering technology, the system realize distributed engineering monitoring in disaster[4].

FBG sensing system is made up of broadband light source module, wavelength demodulation and analysis module, optical channel module, signal processing and data analysis module, power module and grating sensor array. Wavelength demodulation and analysis module consists of optical fiber F-P filter, scanning control circuit and photoelectric detection circuit. Optical channel module consists of optical switch and control circuit. Signal processing and data analysis module consists of A/D sampling, embedded CPU board and software module. Structure chart of FBG sensing system as shown in Figure 4.

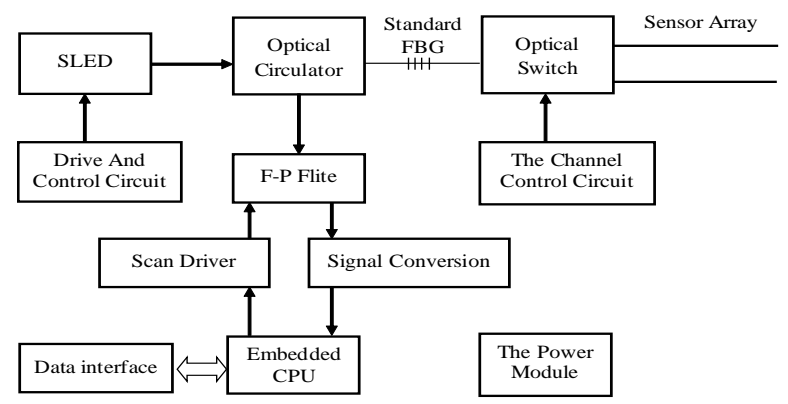

Figure 4. Structure Chart of FBG Sensing System

According to the above measuring principle, the FBG demodulation instrument demodulate the changes of FBG sensor wavelength into the changes of the strain, stress, temperature, displacement. The multiple channels wireless acquisition terminal get the field data, then send data to the receiving server room by GPRS or Beidou. At last the data publishing system process data in real-time and publishing. 


\subsection{System Technical Parameters}

(1) Measurement range and precision of stress:0-5Mpa,5\%;

(2) Measurement range and precision of temperature:- $20^{\circ} \mathrm{C}-+80^{\circ} \mathrm{C}, \pm 0.5^{\circ} \mathrm{C}$;

(3) Measurement range and precision of displacement:0-100mm, $0.5 \%$;

(4) Measurement range and precision of Micro strain: $\pm 1000 \mu \varepsilon, \pm 3 \mu \varepsilon$;

(5) Optical fiber maximum measurement length: 1000m;

(6) The most single fiber measuring points: 30.

\section{Application}

\subsection{Laboratory Calibration}

In the method, the main work of laboratory calibration is metrological verification of measurement parameters(strain, temperature, thrust and displacement). Test methods as follows:

(1) Strain measurement range: $0- \pm 1000 \mu \varepsilon$. The testing process is directly going through test data. The measured object is connected with a set of standard resistance strain gauge, and the measured object is also connected with the measured FBG strain sensor. Comparing the strain data which is detected by the system with standard resistance strain data which is detected by BZ2205C static resistance strain gauge. In the experiment, $\mathrm{K}=526.0389 \mu \varepsilon / \mathrm{nm}$, strain test data as shown in Table 1 .

Table 1. Table of Strain Test

\begin{tabular}{c|c|c|c}
\hline Standard value $(\mu \varepsilon)$ & Measured value $(\mu \varepsilon)$ & Absolute error $(\mu \varepsilon)$ & Relative error $(\%)$ \\
\hline 0 & -0.1 & -0.1 & -0.01 \\
\hline 250 & 251.9726 & 1.9726 & 0.1726 \\
\hline 500 & 498.159 & -1.841 & -0.1841 \\
\hline 750 & 752.3 & 2.3 & 0.23 \\
\hline 1000 & 99.99 & -0.01 & -0.001 \\
\hline
\end{tabular}

Test conclusion: The maximum absolute error is $2.3 \mu \varepsilon$; the maximum relative error is $0.23 \%$.

(2) Temperature measurement range: $-10{ }^{\circ} \mathrm{C}-+65^{\circ} \mathrm{C}$. The testing process is directly going through test data. The measured object is connected with the measured FBG strain sensor. Comparing the temperature data which is detected by the system with temperature data of thermometer. In the experiment, $\mathrm{K}=99.6264^{\circ} \mathrm{C} / \mathrm{nm}$, temperature test data as shown in table 2 .

Table 2. Table of Temperature Test

\begin{tabular}{c|c|c|c}
\hline Standard $\left({ }^{\circ} \mathrm{C}\right)$ & Measured value $\left({ }^{\circ} \mathrm{C}\right)$ & Absolute error $\left({ }^{\circ} \mathrm{C}\right)$ & Relative error $(\%)$ \\
\hline-10 & -10.02 & -0.02 & -0.25 \\
\hline 0 & -0.01 & -0.01 & -0.125 \\
\hline 20 & 20.02 & 0.02 & 0.25 \\
\hline 40 & 39.96 & -0.04 & -0.5 \\
\hline 60 & 59.98 & -0.02 & -0.25 \\
\hline 80 & 79.97 & -0.03 & -0.37 \\
\hline
\end{tabular}


Test conclusion: The maximum absolute error is $-0.04{ }^{\circ} \mathrm{C}$; the maximum relative error is$0.5 \%$.

(3) Thrust measurement range: $0-5 \mathrm{Mpa}$. The testing process is directly going through test data. Comparing the thrust data which is detected by the system with the data of standard stress gauge. In the experiment, $\mathrm{K}=1.9455 \mathrm{MPa} / \mathrm{nm}$, thrust test data as shown in Table 3 .

Table 3. Table of Thrust Test

\begin{tabular}{c|c|c|c}
\hline Standard(MPa) & Measured value(MPa) & Absolute error(MPa) & Relative error(\%) \\
\hline 0 & -0.01 & -0.01 & -0.2 \\
\hline 1 & 0.8786 & -0.1214 & -2.482 \\
\hline 2 & 1.8747 & -0.1253 & -2.506 \\
\hline 3 & 2.8746 & -0.1254 & -2.508 \\
\hline 4 & 3.8383 & -0.1617 & -3.234 \\
\hline 5 & 4.986 & -0.014 & -0.28 \\
\hline
\end{tabular}

Test conclusion: The maximum absolute error is $-0.1617 \mathrm{MPa}$; the maximum relative error is $-3.234 \%$.

(4) Displacement measurement range: $0-100 \mathrm{~mm}$. The testing process is directly going through test data. The measured object is connected with the measured FBG strain sensor. Comparing the displacement data which is detected by the system with the data of vernier caliper. In the experiment, $\mathrm{K}=55.1268 \mathrm{~mm} / \mathrm{nm}$, displacement test data as shown in Table 4.

Table 4. Table of Displacement Test

\begin{tabular}{c|c|c|c}
\hline Standard $(\mathrm{mm})$ & Measured value $(\mathrm{mm})$ & Absolute error $(\mathrm{mm})$ & Relative error $(\%)$ \\
\hline 0 & -0.01 & -0.01 & -0.01 \\
\hline 20 & 19.625 & -0.375 & -0.375 \\
\hline 40 & 39.57 & -0.43 & -0.43 \\
\hline 60 & 59.527 & -0.473 & -0.473 \\
\hline 80 & 79.934 & -0.066 & -0.066 \\
\hline 100 & 100.01 & 0.01 & -0.01 \\
\hline
\end{tabular}

Test conclusion: The maximum absolute error is $-0.473 \mathrm{~mm}$; the maximum relative error is $-0.473 \%$.

\subsection{Engineering Application}

Through the laboratory test, the system is confirmed in accordance with design standards, so we put it into engineering application.

\subsubsection{Introduction of Application Site}

The location of engineering practice is WuChangwan landslide which is located in Fengjie county of the Three Gorges Reservoir Area. Fengjie county is located in the territory of Chongqing City in southwest China, which is one of the key areas of the Three Gorges immigrants, so it has a very important strategic position. WuChangwan landslide is a typical landslide in Three Gorges Reservoir Area. The overall shape of landslide is rectangular, and the landslide go through the central of FengEn highway which is building right now, the front of landslide is next to Daxi River, the terrain slope is $20 \sim 30^{\circ}$, the direction of slope is $140^{\circ}$.

\subsubsection{Testing Method}

FBG monitoring system is suitable for landslide disasters monitoring in the field. Firstly load bearing fiber and FBG multiple parameters sensor are installed in the sliding body, then FBG 
demodulation instrument demodulate the changes of FBG sensor wavelength into the changes of the strain, stress, temperature, displacement. The multiple channels wireless acquisition terminal get the field data, then send data to the receiving server room by GPRS. Finally, the data publishing system process and publish monitoring data.

(1) Design of FBG sensing: The object of engineering application are deep displacement monitoring, internal stress monitoring and surface cracks monitoring, so FBG strain sensor, FBG stress sensor and FBG displacement sensor use to measuring these parameters. Each monitoring site use one optical fiber for measurement and each optical fiber consist of three different kinds of sensor in series.

(2) Monitoring sites arrangement: According to the requirements of geological survey report in this landslide, FBG monitoring system is installed in this landslide, and four monitoring sites are set up, the layout diagram of fiber Bragg grating monitoring sites as shown in Figure 5.

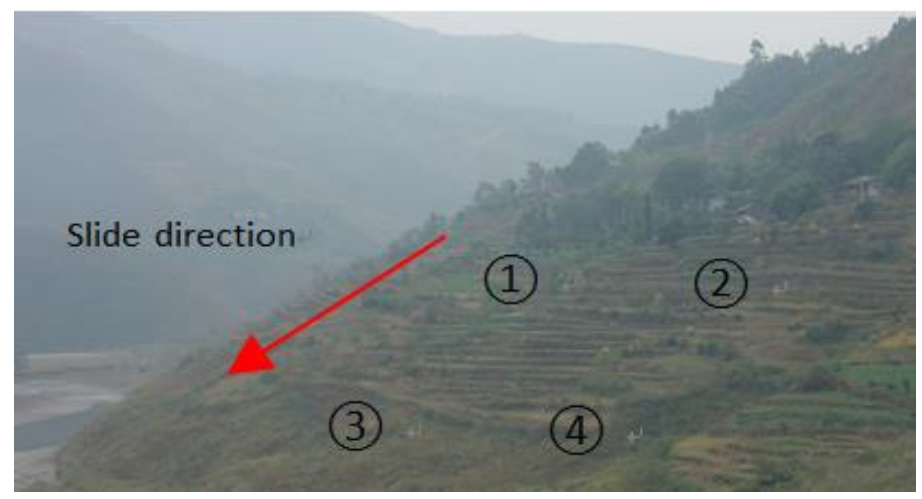

Figure 5. Distribution Chart of FBG Monitoring Sites

The single monitoring site consists of FBG sensing system, multiple channels wireless acquisition terminal and field power supply system, Pictures of FBG monitoring site as shown in Figure 6.

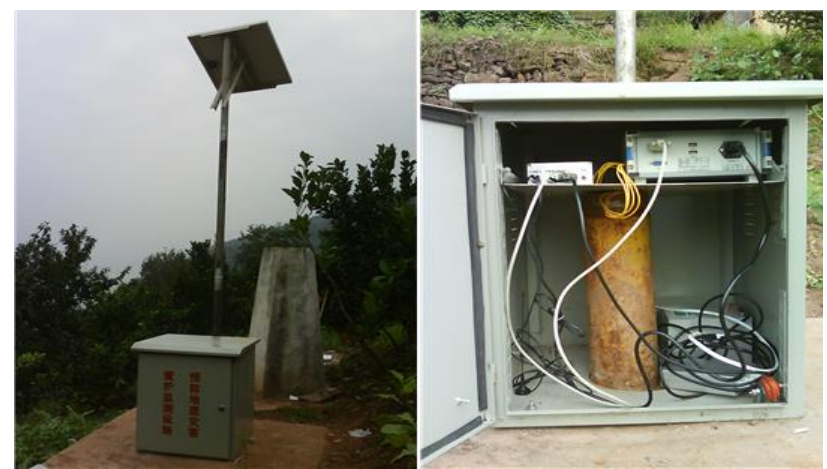

Figure 6. Pictures of FBG Monitoring Site

\subsubsection{Data Analysis and Monitoring Monclusion}

This system have monitored for approximately 12 months, and from January 2012 to December 2012. Firstly, FBG sensors have been buried in 2011 December. When the installation is complete, grout the drilling and do the first test, and check the installation of 
FBG sensors. At the beginning of January 2012, when mud has solidified, finally we finish Sensor data measuring and installation of multiple channels wireless acquisition system.

The monitoring time is from dry season (January to May) to the rainy season (June to September), and then to the dry season (October to December). The system obtains a large amount of monitoring data, the mountain slide trend preliminary judgment. We have preliminary judgment about the sliding trend of mountain inside. We selected some typical stress data of No.1 monitoring site, and the data table as shown in Table 5.

Table 5. Stress Test Table of No.1 Monitoring Site

\begin{tabular}{|c|c|c|c|c|}
\hline Test Time & Difference(pm) & $\begin{array}{c}\text { Variation } \\
\text { value }(\mathrm{pm})\end{array}$ & Stress(Kpa) & $\operatorname{TEMP}\left({ }^{\circ} \mathrm{C}\right)$ \\
\hline 2012-1-4 & 1136 & 0 & 0 & 10.5 \\
\hline $2012-1-20$ & 1157 & 21 & 52.773 & 11.2 \\
\hline $2012-2-4$ & 1169 & 33 & 82.928 & 16.6 \\
\hline $2012-2-21$ & 1160 & 24 & 60.311 & 16.2 \\
\hline $2012-3-4$ & 1156 & 20 & 50.260 & 17.5 \\
\hline 2012-3-18 & 1155 & 19 & 47.747 & 19.9 \\
\hline $2012-4-5$ & 1150 & 14 & 35.182 & 21.4 \\
\hline 2012-4-25 & 1161 & 25 & 62.824 & 20.5 \\
\hline $2012-5-4$ & 1156 & 20 & 50.260 & 23.6 \\
\hline $2012-5-20$ & 1159 & 23 & 57.799 & 24.4 \\
\hline $2012-6-5$ & 1149 & 13 & 32.669 & 28.7 \\
\hline 2012-6-19 & 1176 & 40 & 100.519 & 30.8 \\
\hline $2012-7-10$ & 1186 & 50 & 125.649 & 32.6 \\
\hline 2012-7-15 & 1180 & 44 & 110.571 & 33.2 \\
\hline $2012-7-29$ & 1183 & 47 & 118.110 & 30.5 \\
\hline $2012-8-4$ & 1175 & 39 & 98.006 & 27.1 \\
\hline $2012-8-10$ & 1170 & 34 & 85.441 & 29.5 \\
\hline 2012-8-30 & 1187 & 51 & 128.162 & 29.1 \\
\hline $2012-9-8$ & 1177 & 41 & 103.032 & 28.2 \\
\hline 2012-9-11 & 1181 & 45 & 113.084 & 28.1 \\
\hline 2012-9-10 & 1169 & 33 & 82.928 & 27.4 \\
\hline 2012-9-11 & 1166 & 30 & 75.389 & 22.9 \\
\hline $2012-10-5$ & 1160 & 24 & 60.311 & 19.1 \\
\hline $2012-10-26$ & 1155 & 19 & 47.747 & 17.7 \\
\hline $2012-11-1$ & 1158 & 22 & 55.286 & 15.6 \\
\hline $2012-12-20$ & 1162 & 26 & 65.337 & 11.8 \\
\hline
\end{tabular}

Through the information system of monitoring center, all sensor monitoring data in one year save in the database, and these data could be inquired online. The data curve graph of No.1 stress monitoring site as shown in Figure 7. 


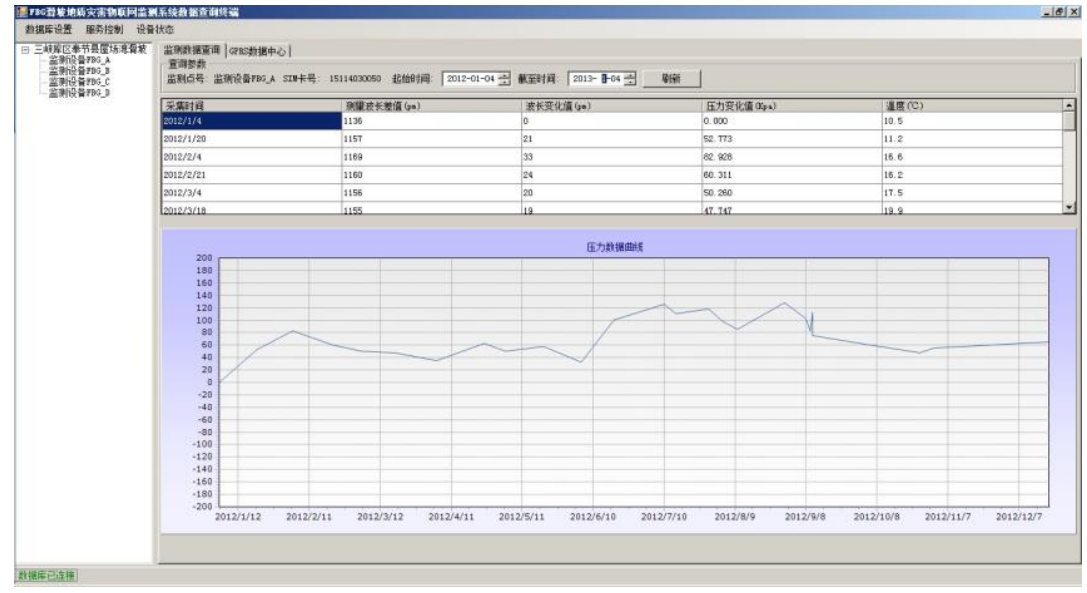

Figure 7. Data Curve Graph of NO.1 Stress Monitoring Site

From the data analysis results, the stress and deformation are not big, landslide is in the state of micro creep. It mainly displays in the change of stress and cracks are small in the dry season, and these parameters have larger changes in the rainy season. So monitoring frequency is increased in the rainy season. During this year, the FBG monitoring system runs stably and obtains a very good monitoring effect, and the system provides a large number of real-time data For geological disaster monitoring and warning. Through a long period of time and multiple sites engineering application, the system is promoted to landslide geological disasters in reservoir bank of the Yangtze river and the Yellow river, and it is also promoted to geological disasters which is caused by earthquake and mine, providing effective technical support for geological disaster prevention and mitigation.

\section{Conclusion}

Using the principle of distributed FBG sensing, the FBG sensor can not only access to space position of sensor installation, but also can get the sensor measurements, realizing the distributed measurement of engineering position and sensor measurement value at the same time. Now we have adopted this theory to develop a prototype, and laboratory calibration and engineering application in the field are completed. Due to its advantages of distributed measurement, high-accuracy and anti-interference, the FBG monitoring system runs stably in the field and obtains a very good monitoring effect. Through further study of optimal placement of the FBG sensor, more accurate assessment of landslide state can be achieved.

The FBG monitoring system provides a new technology, method and equipment for the research of geological disaster engineering monitoring. Through a long period of time and multiple sites engineering application, the next step the system is promoted to the safety monitoring of civil engineering facilities, such as tunnel, cavern, high-rise buildings, hospitals, stadiums, bridges and other special buildings, and to monitor the dangers and to evaluate damage, the FBG monitoring system will have a broad prospect in engineering application. 


\section{Acknowledgments}

This work is supported by the National Natural Science Founds of P.R.China under Grant No. 61327004, and CGS of P.R.China geological survey project No.1212011220169, No.12120113011100.

\section{References}

[1] Optical Fiber Sensors, A. N. Chester, "Kluwer Academic Publishers", Italy (2006).

[2] R. Kashyap, "Fiber Bragg Gratings", Academic Press, (1999), San Diego, USA.

[3] L. Chuanzheng and L. Yanhui, "The paper of geological disaster prevention and geological environment", Journal of Jilin University, vol. 5, (2012), pp. 112-116.

[4] L. Yongqian, Y. Guozhen and Y. Zhi, "Fiber Bragg Grating Sensor.China Acta Photonica Sinica", vol. 11, (2012), pp. 123-127.

[5] G. D. Pitt, P. Extance and R. C. Neat , "Optical-fiber sensors", IEE Proceedings J (Optoelectronics), vol. 132, iss. 4, August (1985), pp. $214-248$.

[6] Z. Ce, C. Wenjun and T. Guoqi, "The research of BHT- II Landslide thrust monitoring system”, The Chinese Journal of Geological Hazard and Control, vol. 3, (2005), pp. 64-69.

[7] M. Prabhugoud and K. Peters, "Modified Transfer Ma-trix Formulation for Bragg Grating Strain Sensors", Journal of Light wave Technology, vol. 2, (2004), pp. 33-39.

[8] B. Culshaw, "Optical Fiber Sensors and Applications", Optics InfoBase Conference, (2004) October 11- 14, Beijing, China.
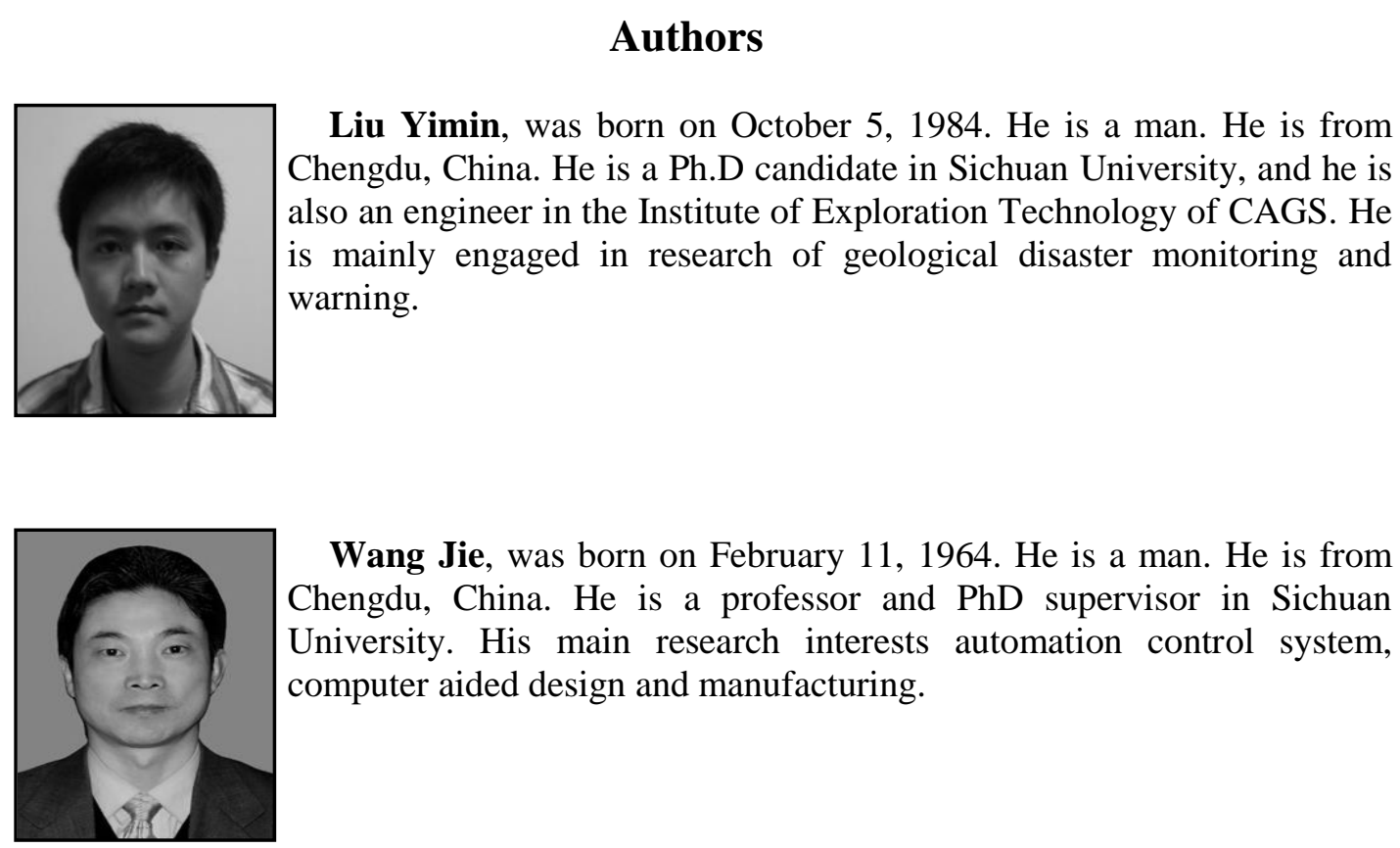

Wang Jie, was born on February 11, 1964. He is a man. He is from Chengdu, China. He is a professor and $\mathrm{PhD}$ supervisor in Sichuan University. His main research interests automation control system, computer aided design and manufacturing.

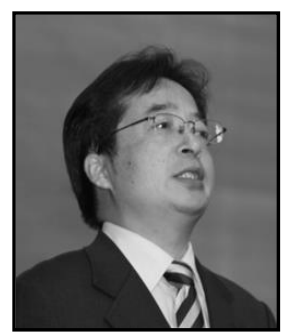

Ji Weifeng, was born on August 8, 1961. He is a man. He is from Chengdu, China. He is a professorate senior engineer in the Institute of Exploration Technology of CAGS. 


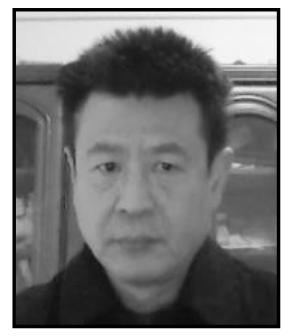

Zhou Ce, was born on June 10, 1965. He is a man. He is from Chengdu, China. He is a professorate senior engineer in the Institute of Exploration Technology of CAGS.

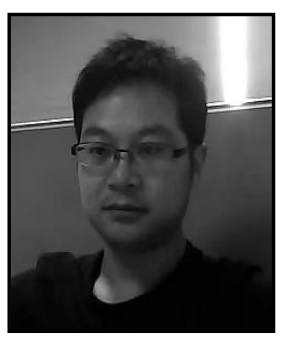

Chen Wenjun, was born on December 23, 1977. He is a man. He is from Chengdu, China. He is a senior engineer in the Institute of Exploration Technology of CAGS. 
International Journal of Control and Automation Vol.7, No.10 (2014) 